\title{
Le rêve matawinien de l'abbé Provost
}

\section{Christian Morissonneau}

Volume 54, 1987

Culture et religion dans la région de Lanaudière

URI : https://id.erudit.org/iderudit/1006962ar

DOI : https://doi.org/10.7202/1006962ar

Aller au sommaire du numéro

Éditeur(s)

Les Éditions Historia Ecclesiæ Catholicæ Canadensis Inc.

ISSN

0318-6172 (imprimé)

1927-7067 (numérique)

Découvrir la revue

Citer cet article

Morissonneau, C. (1987). Le rêve matawinien de l'abbé Provost. Sessions d'étude - Société canadienne d'histoire de l'Église catholique, 54, 55-67. https://doi.org/10.7202/1006962ar
Résumé de l'article

L'abbé Provost a été le précurseur du curé Labelle. Son projet matawiniendont il définit les grandes lignes dès 1862 s'inscrit dans la mêmeinspiration, la même logique, la même stratégie. Il a été le véritable pionnierde l'idéologie nordiste.
Tous droits réservés @ Les Éditions Historia Ecclesiæ Catholicæ Canadensis Inc., 1987
Ce document est protégé par la loi sur le droit d'auteur. L'utilisation des services d'Érudit (y compris la reproduction) est assujettie à sa politique d'utilisation que vous pouvez consulter en ligne.

https://apropos.erudit.org/fr/usagers/politique-dutilisation/ 


\title{
Le rêve matawinien de l'abbé Provost
}

\author{
Christian MORISSONNEAU \\ Département de géographie \\ Université du Québec à Montréal
}

\section{RÉSUMÉ}

L'abbé Provost a été le précurseur du curé Labelle. Son projet matawinien dont il définit les grandes lignes dès 1862 s'inscrit dans la même inspiration, la même logique, la même stratégie. Il a été le véritable pionnier de l'idéologie nordiste.

Le volume que publie l'abbé Provost, en 1883, sorte de bilan des années de campagne sinon de croisade colonisatrice, ainsi que son journal manuscrit, présentent explicitement un projet de colonisation, peut-être équivoque. ${ }^{1}$

À l'origine du mouvement de colonisation matawinien, on trouve, comme en tous les mouvements colonisateurs du Nord, un prêtre. L'œuvre de ce curé mérite d'être connue autant par son activité dans le développement agricole que dans le développement global du nord de Joliette. L'abbé Provost n'a pas laissé la marque prestigieuse maintenant légendaire du curé Labelle, mais son œuvre aussi vaste par sa vision s'inscrit dans la même inspiration, la même logique, la même stratégie. Dans le temps, cette œuvre précède celle de Labelle. La mythique du Nord nourrit les discours et le projet matawinien huit ans avant que le curé Labelle n'arrive à Saint-Jérôme. Provost n'a que vingt-cinq ans lorsqu'il regarde vers le Nord et $\mathrm{y}$ voit un territoire à occuper.

Théophile-Stanislas Provost est né le 31 juillet 1835 à Verchères. Il étudie au collège de Saint-Hyacinthe et au séminaire de Montréal, puis de-

1 Th. S. Provost, La Bourse et la vie, Joliette, Imprimeric du Collège de Joliette, 1883. 
vient chapelain de la première prison de réforme de la province, à l'île aux Noix (1858). Deux ans plus tard, il est nommé curé de Saint-Alphonse Rodriguez, dans le comté de Joliette.

Je commençai alors à m'occuper de colonisation. Je me trouvais dans ces terres nouvelles au milieu de colons... ${ }^{2}$

Il se consacre à l'ouverture d'une paroisse plus au nord: Saint-Côme. Il vise plus grand et, dès 1862 , définit ce que nous pouvons appeler le projet matawinien, projet nordique exemplaire. Cette même année, il vante, dans ie journai Le Colonisateur, «les cantons du Nord». La même année, il fait un discours remarqué devant la Société de colonisation de Joliette. Il souhaite faire l'exploitation de «la belle plaine qui repose au pied de ces montagnes au milieu de laquelle la rivière Mantawa coule...». La région que nous nommons Matawinie, mieux que la Mantawa ou la Mantavaisie, employées indifféremment par les prêtres du temps, entre dans l'histoire de la colonisation.

Assurément, des compagnies avaient, depuis les années 1850, ouvert quelques chantiers au nord de la Matawin et se servaient de la rivière pour le flottage et comme voie de pénétration à partir du Saint-Maurice (au poste de Matawin), mais il ne s'agissait que de points d'exploitation sporadique, sans occupation permanente, avec infrastructures spécialisées et réduites au minimum. L'entrepreneur de chantier (le jobbeur), travaillant pour la compagnie, représente une quelconque autorité; pas de familles, pas de prêtres, seul l'ordre du travail. Le curé Provost est le premier à parler de la région, à en faire l'éloge, à vouloir en faire la conquête, l'occupation, le développement, c'est-à-dire à la coloniser, et surtout à lui donner signification et à la faire entrer dans l'«histoire».

La colonisation de la Matawinie s'inscrit dans le grand mouvement de conquête territoriale nordique, mais l'abbé Provost est peut-être le premier leader québécois à proposer une stratégie globale, un projet aux multiples traits et significations qui en font un mouvement original. Dès 1860 , le prêtre s'intéresse à la colonisation du nord de Joliette. Il détermine le site de Saint-Côme qu'il ouvrira quelques années plus tard. Il se lance dans la propagande matawinienne, en termes pleins de la grandeur, de l'enthousiasme et de l'optimisme du livre de Rameau, publié en $1859^{3}$. Provost a-t-il lu $L a$ France aux colonies? A-t-il connu des correspondants de l'essayiste français? Ses discours et écrits de propagande sont empreints du même message. Le discours qu'il a fait à Joliette en 1862 demeure le premier contenu de parole québécois à donner une signification multiple à l'expansion vers

2 Journal de Provost.

3 Edme Rameau de Saint-Père, La France aux colonies, Paris, 1859. 
le Nord. Pourtant, quand le curé Labelle préface La Bourse et la vie en 1883, il ne mentionne pas que le véritable pionnier de l'idéologie nordiste c'est Provost, l'auteur du livre. L'ouverture de la Matawinie est un des meilleurs exemples de la colonisation du Nord. Provost en a été le penseur, l'organisateur, le donneur de sens. Pour lui, la conquête du territoire matawinien est un phénomène global. Ce projet du curé Provost est beaucoup trop riche de significations pour se laisser réduire en une instance. Notre analyse, à partir des écrits du prêtre, vise à retrouver la logique interne qui en donne cohérence. Elle veut éclairer le mouvement de colonisation qui suivra. On peut y dégager trois composantes principales: une stratégie géopolitique, un projet religieux, un développement économique par étapes.

\section{Une stratégie géopolitique}

Le projet de l'abbé Provost est d'abord une stratégie géopolitique. C'est elle qu'a proposée Rameau et que répéteront les constructeurs du mythe du Nord; quand ils parlent de Terre promise, c'est une géopolitique mythifiée qu'il faut reconnaître: l'occupation du sol, à la fois pour l'expansion territoriale et la conservation de la population. Dans les années 1860, non seulement la culture de la collectivité canadienne-française est mise en question, mais cette collectivité elle-même, menacée d'assimilation par les départs massifs des migrants vers les États-Unis, demeure fragile. Les Canadiens français n'occupent qu'une faible partie du territoire provincial: il leur faut accroître le territoire national, agrandir le domaine ancestral par l'occupation du sol. Il s'agit en premier lieu d'une riposte au défi du rapport Durham et à l'émigration. Si l'Anglais cherche l'assimilation, la stratégie consiste à échapper à son influence en s'éloignant des terres et des villes de la vallée laurentienne pour se grouper, se fortifier et se conserver «à l'abri des montagnes», dans l'isolement relatif. Coloniser signifie alors occuper le sol pour assurer l'implantation de la «race» et le développement d'un territoire qui appartienne au groupe. Le discours de Provost qui allait attirer le curé Brassard à l'œuvre colonisatrice présente clairement la situation et la stratégie proposée.

$\mathrm{Si}$ donc nous voulons rester ce que nous sommes déjà et nous agrandir comme peuple dans la proportion de nos voisins du Haut Canada, si nous voulons rompre le blocus que l'on a déjà commencé contre nous dans notre Bas Canada et ne point nous laisser entasser sur les rives du grand fleuve pour y périr d'un seul coup, attachons-nous au sol avec ardeur ... Mais il est un autre terrain, messicurs, bien autrement important ... C'est le versant septentrional des Laurentides et la belle plaine qui repose au pied de ces montagnes au milieu de laquelle la rivière Mantawa coule... ${ }^{4}$

4 Journal de Provost. 
Le projet matawinien de colonisation trouve ici la part la plus importante de sa signification. Il en est ainsi de la colonisation nordique québécoise. Ce projet est déjà, un siècle plus tôt, appliqué au territoire, le «maître chez nous» des années 1960.

Le Nord québécois est la Terre promise réservée au développement du Canada français. Provost délimite quelle fraction nordique doit être le premier enjeu.

En jetant les yeux sur la carte des territoires du Nord ... Le quarante-neuvième parallèle de latitude au nord, la rivière des Outaouais et le Saguenay à l'ouest et à l'est, voilà les limites qui doivent circonscrire notre action colonisatrice...5

La configuration montagneuse ou à tout le moins de collines des régions nordiques du Québec, telles que définies par Provost, alliée à leur immensité et virginité aide à la naissance d'un mythe territorial chez un peuple en crise socio-politique. Les Laurentides forment une longue zone plus ou moins accidentée qui stimule le géostratège même si elle peut repousser le colon éventuel. Jusqu'alors, le québécois n'habite pas les hauts reliefs; il est tantôt cultivateur des vallées ou des plaines du grand fleuve, tantôt coureur de continent, mais jamais il n'accroche sa maison et n'ouvre des sillons sur les pentes des montagnes. Mais la montagne protège, c'est le particularisme conservé; la plaine est ouverture au monde, c'est la proximité obligée avec l'autre, avec l'étranger ou l'ennemi. Provost cite comme exemplaires les peuples qui ont résisté à l'invasion en se réfugiant dans les montagnes difficilement accessibles, entre autres, les gens du Montenegro repoussant l'influence turque. L'esprit des patriotes de 1837 n'est pas mort. Pour le prêtre, la montagne assure aussi refuge à la foi, comme elle l'a fait en Écosse, au Tyrol, dans les Cévennes. Elle protège d'abord l'ethnie et la fortifie, militairement et moralement.

Qui sait si le peuple canadien ne sera pas trop heureux un jour d'aller chercher un asile dans ces montagnes ou de cacher derrière elles les restes de sa nationalité? Les guerres et les persécutions font monter les hommes vers les hauts lieux. Et puis quelles populations ont plus d'énergie et de force que celles des montagnes? ... II y a du roc dans les âmes comme dans la nature. ${ }^{6}$

Le curé Labelle également et les tenants du Nord comme Terre promise insistent à la même époque sur la montagne-refuge, c'est-à-dire sur les Laurentides comme région protégée et protectrice, sans occupation étrangère et ne pouvant l'être. Rameau pour sa part parle du froid comme de l'élément géographique le plus répulsif à l'étranger, élément avec lequel

5 Provost, La Bourse, p. 161-162.

6 Ibid., p. 166. 
le Canadien français entretient une longue familiarité et adaptation. La Matawinie, géographiquement, selon Provost, peut tenir cette promesse du territoire refuge, terre d'élection. Quand on atteint l'actuel village de Sainte-Émélie-de-l'Énergie, venant du Sud, on peut effectivement voir la montagne comme une barrière au-dessus de la plaine. La route emprunte un passage étroit dans la gorge qu'a tracée la rivière Noire. Provost et les contemporains ne pouvaient pas ne pas voir l'image de fortifications, à tout le moins de défense, que suggère ce paysage accidenté. Les vallées de la Matawin et de la rivière Sauvage, entre autres, rompent les élévations et permettent une vision d'établissement humain important. Le projet matawinien s'inscrit parmi les désirs d'extension territoriale comme en ont divers peuples dans l'histoire, en même temps que d'expansion nationale et de quasi-repli. L'ouverture de la Matawinie est une stratégie géopolitique, à la fois par le contexte socio-politique et la situation et configuration géographiques des lieux choisis?.

Nos montagnes pourraient être aussi un jour un boulevard de liberté, une barrière infranchissable à toute armée qui envahirait notre pays: habitées et cultivées elles seraient notre salut, car nous y pourrions puiser les ressources nécessaires. Les populations groupées en arrière continueraient d'y vivre en pleine sécurité ... il est donc rationnel de chercher à s'assurer un agrandissement de territoire dans des endroits où l'indépendance et la nationalité canadiennes seront toujours à l'abri. ${ }^{8}$

La colonisation est le premier projet nationaliste explicite après la rébellion de 1837 .

\section{Un projet religieux}

Après avoir retrouvé, dans la logique interne du mouvement, le projet géopolitique, l'analyse du discours et de l'œuvre du curé Provost nous révèle l'aspect religieux qui donne au projet global la plus grande part de sa spécificité. Au moins deux mouvements de population, à l'époque moderne, peuvent se comparer à la colonisation nordique: l'installation des Boers au Transvaal en Afrique du Sud et celle des Mormons dans le Grand Bassin de l'Utah, aux États-Unis. La dimension verticale est celle qui dynamise, qui donne sens: le Transvaal attirait peu, encore moins les environs

7 Tout le XIXe siècle est plein d'une littérature de géographes et de pseudo-géographes où géographie égale déterminisme biophysique. On a tiré de ces déterminismes des doctrines géopolitiques appuyées sur la supposée adéquation entre des ethnies et des configurations territoriales ou des directions géographiques privilégiées. La géopolitique allemande portera ces idées à leur point extrême, sinon absurde, à l'époque nazie. (Le général Karl Haushofer et l'Institut für Geopolitik)

8 Provost, La Bourse, p. 167. 
du lac Salé, pas plus les vallées centrales des Laurentides. Une géographie de l'esprit serait signifiante dans ces régions plus ou moins répulsives, dont les traits biophysiques sont précisément transcendés par l'espérance d'un groupe d'hommes. Le mythe du Nord peut se comprendre comme message messianique. Le mouvement qui en est issu prend une autre signification que les mouvements de population analogues de la même époque. Ainsi, la marche vers l'Ouest des Mormons n'obéit pas au même dessein que celui des pionniers du temps s'enfonçant dans le Grand Désert américain vers les Rocheuses. La même opposition s'obscrvc lorsquie l'ôn compare les colonisations nordiques québécoise et ontarienne, dans les Clay Belts9.

Le curé Provost doit être vu, en tant que prêtre, comme un leader religieux. Il rapproche sa vision de la Matawinie de celle d'autres régions déjà mentionnées, refuge à la persécution religieuse: les Cévennes pour les protestants français, les Highlands d'Écosse pour les catholiques écossais. Pour Provost, la «nationalité» canadienne-française s'achemine vers l'assimilation, c'est-à-dire la perte de son originalité culturelle, entre autres «la langue et la foi». Il est du devoir des leaders naturels de ce peuple catholique d'orienter son avenir. Spirituellement et non seulement matériellement, le présent inquiète. Beaucoup de nationaux partent vers le domaine territorial des Anglo-Saxons protestants, où la présence étrangère détériore l'esprit et la foi ancestrale. Plus encore, ces mêmes Anglo-Saxons protestants occupent les Cantons de l'Est, dictent leur vision du monde et imposent leur conception du développement dans les villes, s'infiltrent au pied et dans les contreforts des Laurentides (ainsi les établissements de New Glasgow, Rawdon, Chertsey, dont les appellations, ainsi que celles des cantons, sont un défi au patrimoine français). Ce sont des étrangers, à la langue et surtout à la foi étrangère. Les Laurentides ne peuvent abriter les temples d'une religion hérétique. À New-Glasgow et Radstock, aux noms de lieux presque agressifs en territoire français, il faut opposer les Saint-Micheldes-Saints, les Saint-Zénon, les Saint-Côme. La croisade commence. Il faut planter des croix et ouvrir des paroisses aux quatre coins du Nord, comme des bornes immuables et des témoins de la présence canadienne-française en ce territoire qui deviendra et restera français et catholique. Le curé-colonisateur Provost agrandit le territoire national; il agrandit en même temps le territoire catholique, en plein contexte québécois de mission. Le projet

9 Voir: A. Gosselin et G.P. Boucher, Settlement Problems in Northwestern Quebec and Northeastern Ontario, Canada, Department of Agriculture, Publ. no 758, Technical Bulletin 49, 1944; G. McDermott, «Frontiers Settlement in the Great Clay Belt, Ontario and Quebec», Annals of the Association of American Geographers, 51 (1961), p. 261-273; aussi, P. Biays, Les marges de l'cekoumène dans l'est du Québec, Québec, P.U.L., 1964, p. 276 (Travaux et documents du Centre d'études nordiques). 
matawinien s'explique en terme de contre-réforme, en terme anti-protestant. Le missionnaire-colonisateur oppose, au Sud anglo-protestant, le Nord franco-catholique. Quelques années plus tard, Labelle et les Jésuites rêvent de faire de Nominingue une capitale religieuse; Provost fera de la Matawinie une région «où souffle l'esprit».

Pour comprendre la profondeur du fait religieux dans la conquête matawinienne, il nous faut le replacer dans le sens messianique chrétien traditionnel. Le christianisme est apparu, s'est diffusé et s'est conservé par les miracles. Une des croyances fondamentales des chrétiens demeure que l'homme est déjà changé par l'incarnation et la résurrection. L'homme porte en lui l'homme nouveau et la terre nouvelle. Ainsi s'expliquent les mouvements messianiques et millénaristes d'inspiration chrétienne. Cette croyance est plus ou moins vive selon les époques; elle réapparaît souvent, exubérante sinon violente, aux périodes de crise. Au XIX ${ }^{\mathrm{e}}$ siècle, de nombreux prêtres québécois surent la ranimer et l'entretenir. La parole mythique retentit dans les églises et les salles de conférences. Des clercs et des intellectuels rallument le principe «Espérance» ${ }^{10}$. On ne comprend pas la colonisation québécoise sans la rattacher à ce messianisme qui ressurgit sporadiquement dans les sociétés menacées d'acculturation, surtout religieuse. Ouvrir la Matawinie s'entend comme projet d'espérance, comme réaction contre-acculturante. Il ne s'agit pas d'œuvre mystique comme certains chercheurs l'expriment. La mystique est une voie pour parvenir à l'union avec Dieu, à tout le moins une expérience de cette union; la foi est la vertu première. Dans le mouvement colonisateur québécois, c'est l'espérance qui dynamise; l'affect prime. Les régions conquises ne prennent et gardent vie sociale qu'avec une alimentation permanente en irréalité, en informatique mythique. C'est une explication de l'apparente irrationalité des décisions des missionnaires de faire s'enfoncer les colons dans la sauvagerie montagneuse, l'isolement, la «terre de roche». Seule cette logique religieuse donne sens à ce projet et ce mouvement en apparence insensés.

Comme le dit Provost, l'espérance est la nourriture de l'âme. Mais la parole d'espoir peut voiler des intérêts plus matériels... Il demeure en tout prêtre et chef de paroisse, celui qui veut garder contrôle et conserver un pouvoir temporel. Des prêtres ont pu voir à ce que l'autorité cléricale se conserve intacte dans des régions neuves isolées de la ville et de ses dangers de dissolution, de dégradation et d'irréligion. Le prêtre songe sans doute à accroître le nombre de ses ouailles, du moins à ne pas le laisser diminuer puisqu'il est aussi pasteur. L'essentiel du projet est d'animer la col-

10 Voir l'œuvre quasi entière de Ernst Bloch, appuyée sur une philosophie matérialiste de l'espérance; il développe une longue argumentation à la fois philosophique et empirique dans un ouvrage captivant: Le principe espérance, t. I, parties 1, 2, 3, Paris, Gallimard, 1976. 
lectivité canadienne-française, lui donner sens et la nourrir psychiquement. Le curé redit la mission de la nation, dont il fait une nation théophore, à l'image de la nation juive dispersée.

\section{Un développement économique par étapes}

La colonisation, en Matawinie comme ailleurs au Québec, débute par la conquête du sol. Les idéologues du mouvement utilisent une phraséologie agraire mais la plupart ajouitênt, à là loúange agricule du territoire, des idées de développement minier et industriel.

Provost, d'emblée, en énumérant le potentiel de la région, dépasse la perspective agricole. Implicitement s'esquisse un plan de développement par étapes, qui tirera profit au maximum des ressources. Le titre du livre n'est-il pas tout un programme: La Bourse et la vie?

À tous points de vue, à tous égards, sous le rapport des richesses naturelles, de la fécondité du sol, des établissements industriels à y faire, le territoire de la Mantavaisie est avantageux et mérite d'attirer l'attention de ceux qui s'intéressent à la cause de la colonisation. ${ }^{11}$

Ce territoire refuge et lieu sacré, que faut-il pour qu'il enrichisse matériellement ses nouveaux habitants?

Cette région d'une immense étendue n'a besoin que de la main de l'homme et de quelques années pour devenir l'égale des plus florissantes parties du Canada. ${ }^{12}$

Pour atteindre cette réussite, la «main de l'homme» sera d'abord celle du défricheur, puis celle du cultivateur; plus tard celle de l'homme d'affaires. Une stratégie de développement s'esquisse qui voit le défrichement, donc les prémices agricoles, comme première étape. Le défricheur est le héros de l'épopée du Nord, c'est lui qui ouvre la route et le prêtre lui montre le chemin. Les définisseurs de la colonisation québécoise s'entendent sur cette dualité: c'est avec la croix et la hache que la civilisation pénètre dans la sauvagerie, et l'ouverture de la Matawinie se fait d'abord par le recul de la forêt et la conquête du sol. Le défricheur transforme le désert: de nature celui-ci devient culture.

On aurait pu croire, il y a vingt-ans, qu'un obstacle insurmontable s'opposait à la réalisation de ce beau rêve dans la chaîne de montagne qui semble barrer le passage vers le nord ... Oui, la hache du défricheur finira par percer graduellement la chaîne des Laurentides, elle n'arrêtera son œuvre que sur les bords de la Mantawa ... La colonisation du grand Nord sera un immense trésor pour toute la province. ${ }^{13}$

\footnotetext{
11 Provost, La Bourse, p. 140.

12 Ibid., p. 162.

13 Ibid., p. 164-165.
} 
Provost décrit sa région de façon attirante, assuré qu'elle est fertile dans sa plus grande superficie; assuré ainsi qu'il faut commencer par garantir la subsistance, vieille réaction de bon sens que l'homme doit penser se nourrir, c'est-à-dire exploiter le sol avant d'élargir son activité d'exploitation illimité. Le discours matawinien participe ainsi du même esprit que celui, à la même époque, du lac-Saint-Jean, de la vallée de la Matapédia, de la région de Labelle ou des hauteurs appalachiennes.

Mais on est loin de l'agriculture-résignation, de l'agriculture destinée à ceux qui sont «nés pour un petit pain». L'agriculteur s'enrichit par le travail. C'est en effet en termes de réussite matérielle et d'argent que Provost parle. Le défricheur est un conquérant, plein d'initiatives, non un bucolique. Provost ne décrit pas le cultivateur comme certains tenants du retour à la terre. C'est au sens des affaires de cet homme, à son éthique du travail, à son aspiration à la richesse, qu'il en appelle. Pas de rappel à la frugalité comme valeur terrienne, mais à la prospérité par l'agriculture. L'accumulation des termes et qualificatifs économiques ne laisse pas de doute sur le modèle agricole visé dans le paragraphe suivant:

Le succès est infaillible, surtout si c'est un fils de cultivateur qui se dirige à Mantawa ... Le terrain partout est facile à cultiver, et moyennant économie et bon travail, il paiera jusqu'au centuple les peines du colon. Le prix des terres n'est rien; il n'est généralement que de trente centins l'acre, en sorte que pour la bagatelle de trente piastres, payées au Gouvernement dans l'espace de cinq ans un colon devient propriétaire d'un lot de cent acres... ${ }^{14}$

La fin du XIX $\mathrm{X}^{\mathrm{e}}$ siècle, au Québec, est l'époque du capitalisme sauvage: dans des villes québécoises, comme Valleyfield, Drummondville ou Trois-Rivières, des manufactures captivent des ruraux déracinés. L'exode rural se poursuit vers les villes du textile américaines. Des crises économiques font douter de l'harmonie de la croissance industrielle.

La plupart des économistes du temps pariaient encore sur l'agriculture pour prévenir les aléas du développement industriel. Provost consacre des pages dithyrambiques à l'état de l'agriculteur et fustige, comme ses confrères en colonisation, l'attirance pour les professions libérales encombrées et le travail débilitant dans les «facteries» de la Nouvelle-Angleterre $^{15}$. Les professions libérales représentent des éléments parasites dans une petite société, des éléments non productifs en trop grand nombre chez un peuple encore pauvre.

14 Ibid., p. 181-182.

15 En termes autres: d'un côté, l'ouvrier qui n'a que sa force de travail à vendre à l'étranger, de l'autre, le colon qui contrôle ses moyens de production. 
L'agriculture n'est-elle pas un art, la mère même des arts, comme le dit Xénophon? ... Nouveau Moïse, nouvel Enoch, littéralement «il marche avec Dieu». Oh! qu'on a tort dans une infinité de familles canadiennes-françaises de se déclasser comme on le fait! On prive d'une belle vie nombre d'enfants que l'on pousse dans des professions libérales ou autres carrières ... La terre cultivée est à la fois la plus abondante des mines et le plus solide de tous les biens. C'est pour cela que l'économie rurale est à la base de la richesse des États et des particuliers. ${ }^{16}$

La philosophie agrarienne de l'élite politique et intellectuelle améri-

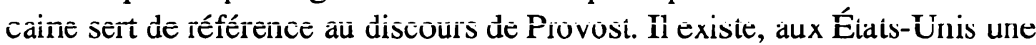
tradition anti-étatique et anti-urbaine entretenue depuis les temps légendaires des Pionniers puritains. Cette tradition est représentée aussi bien chez les écrivains à la recherche de la «prairie perdue» que chez les politiciens les plus vénérés des XVIII et $\mathrm{XIX}^{\mathrm{e}}$ siècles, ce qui n'est pas la moindre contradiction dans ce pays symbole du progrès industriel illimité et de l'urbanisation galopante. L'abbé Provost remémore, pour les lecteurs, le souvenir des hommes d'État et princes qui s'honoraient de leur intérêt pour l'agriculture, de Henri IV de France à George III d'Angleterre en passant par Edmund Burke et Olivier de Serres. Des pages entières sont consacrées à la compilation des grands noms qui, depuis l'Antiquité, ont donné caution à l'activité agricole. Provost étale là un riche référentiel rarement rencontré dans les écrits des colonisateurs, plus sobres d'ordinaire quant aux antécédents philosophiques et historiques. Dans La Bourse et la vie, l'auteur insiste aussi sur les voisins du Sud: depuis Washington retoumant dans sa ferme de Mount Vernon, Jefferson l'idéologue agrarien et Jackson le symbole du pionnier, jusqu'aux contemporains des années $1870^{17}$. La pensée «ruraliste» de Labelle et de Provost ne doit donc pas être cataloguée réactionnaire; elle s'appuie sur le même système d'idées que celui de l'élite américaine de l'époque qui privilégie le «homesteader», libéré par le travail de la terre et par la propriété terrienne. Pour en comprendre le fondement, il faut saisir cette pensée en termes moraux. La rationalité de ces hommes du XIX $\mathrm{X}^{\mathrm{e}}$ siècle s'exprime en objectifs éthiques et qualitatifs plus qu'en objectifs de productivité et quantitatifs. Comme nous l'avons mentionné, les Américains agrariens, eux aussi pénétrés du mythe-utopie de la Terre promise, pénétrés aussi de l'esprit biblique, connaissent la malédiction divine sur la ville, lieu de l'enracinement de Caïn, «errant dans la terre de Nod; et Caïn n'a plus de lieu, ni humain ni géographique, parce que le meurtre détruit le lieu ... Il va s'enraciner autrement, se fixer pour se défen-

16 Provost, La Bourse, p. 28-31.

17 On ne connaît pas assez les courants de pensée chez nos voisins du Sud: à la même époque d'ardeur idéologique colonisatrice québécoise, une large fraction à l'élite intellectuelle et politique américaine rêvait d'une société rurale idéale. Voir l'ouvrage de Hofstadter, The Age of Reform. 
dre, non plus comme agriculteur mais comme fondateur de ville» ${ }^{18}$. La ville, c'est l'endroit où Caïn sera lui-même, à l'abri du regard divin. La ville, le lieu de l'homme sans Dieu.

Tout en partageant les vues des idéologues américains sur l'idéal ruraliste, Provost, sans contradiction apparente dans son discours, accepte aussi les idéaux de l'entrepreneur américain, croyant au progrès sans limite par l'exploitation intégrale du pays et par le développement technique. Les plus remarquables missionnaires-colonisateurs du mouvement nordique, l'abbé Provost en Matawinie, le curé Labelle dans le nord de Montréal, dans les années 1870-1880, l'abbé Ivanhoë Caron en Abitibi, en 19101920 , ont en commun la même idée du développement économique étapiste.

Provost recherche les voies qui assureront la sauvegarde de la collectivité, il souhaite un développement indigène. Son nationalisme économique ira jusqu'à choisir et appuyer un candidat conservateur pour les élections fédérales en 1891; il trace le programme et écrit les discours car, selon son journal, la pensée conservatrice vise à une politique nationale. Le principe libéral de la réciprocité de commerce avec les États-Unis est une «porte ouverte à l'annexion et une menace à la foi catholique». Provost lance, dès les années 1880 , le slogan «maitres chez nous».

Provost ne condamne pas la ville. Elle peut être le lieu de l'homme qui réussit matériellement et le lieu de l'homme avec Dieu. Joliette fixe, pour lui, comment, de la sauvagerie, naît une cité, fruit du labeur et du commerce des hommes, sous la bienveillante lumière cléricale. Une agriculture prospère et de bons moyens de communication: la ville a sa place; elle facilite les échanges, diffuse le savoir et lance les entreprises, entretient l'émulation et l'innovation.

Où était la ville de Joliette il y a un demi-siècle! Une quasi-solitude au sein de laquelle germait un avenir de prospérité ... Les couvents se multiplient et s'agrandissent. Ils suivent, sous l'impulsion divine, la marche génerale du vrai progrès ... Des hommes de dévouement sont aujourd'hui comme autrefois à la tête des affaires ... Joliette peut être appelée avec raison, et est effectivement la ville du grand Nord ... vingt paroisses y apportent leurs produits ... des milliers de personnes ... viennent ce jour-là vendre ou échanger leurs produits ... continuer, accentuer, accélérer je dirais même, le mouvement général de progrès qui s'y constate de jour en jour. ${ }^{19}$

En 1870, Provost fait le tracé d'un chemin qui partirait de Saint-Michel et passerait à l'ouest du Lac Bourget (lac Ouareau actuel), vers le

18 J. Ellul, Sans feu ni lieu: signification biblique de la grande ville, Paris, Gallimard, 1975.

19 Provost, La Bourse, p. 35-38. 
«Nord de Montréal» et lance l'idée d'un chemin de fer de Joliette à SaintJean-de-Matha puis à Sainte-Émélie-de-l'Énergie.

La quatrième étape est celle du chemin de fer; le développement industriel suit ces étapes. Provost sollicitera sans cesse, mais en vain, le gouvernement fédéral à continuer le chemin de fer jusqu'à Sainte-Émélie-de-l'Énergie car, en 1892, le gouvernement fédéral octroie 3200 \$ par mille, pour une distance de huit milles. Un ingénieur du Canadian Pacific Railway fait même le tracé avec le prêtre jusqu'à Sainte-Émélie. Le chemin de fer est pour lui, à partir de Joliette, comme pour Labelle, à partir de Saint-Jérôme, la panacée au sous-développement. Il faut construire les rails à partir de ces villes-frontières du Nord et l'arrière-pays s'ouvrira, la conquête, l'occupation et le développement seront assurés. Les dernières décennies du XIX ${ }^{\mathrm{e}}$ siècle, pour les optimistes du progrès, appartiennent à l'épopée du rail: les rubans d'acier traversent les continents, aux États-Unis comme en Russie et au Canada. Arthur Buies s'exclame lyriquement devant le chemin de fer du Lac-Saint-Jean, en construction ${ }^{20}$, Provost, devant celui qui doit se construire vers la Matawinie.

Mais ce qu'il faudrait pour diriger rapidement un fort courant de colonisation dans ces lieux ... ce serait un chemin de fer ... N'entendrons-nous jamais le cri strident d'une locomotive soulever les échos endormis de ces grandes solitudes? Quand verrons-nous une machine à vapeur traîner de longs chars de freight et de passagers à travers les Laurentides et se promener triomphalement dans la vallée de Mantawa ... De grandes richesses, d'inépuisables ressources sont à la portée des capitalistes. Que ces capitalistes soient le gouvernement ou quelque compagnie, un particulier même, peu importe. ${ }^{21}$

Le projet de colonisation s'entend alors comme l'entendaient les Européens lorsqu'ils décidaient d'exploiter une région africaine ou asiatique, surtout lorsqu'ils y détachaient des nationaux pour ce faire. Les colons, comme on les appelait, se faisaient planteurs si les conditions pédo-climatiques étaient bonnes ou exploitants miniers et industriels selon les ressources. Pas de bornes dans l'exploitation, la terre appartient à celui qui l'exploite le mieux (selon les règles et normes européennes!) Même phénomène au Québec. Les premiers colons seront certes des défricheurs, mais l'exploitation ne se limitera pas au sol, elle visera aussi bien et en même temps la forêt et le sous-sol, puisque la nature est inépuisable et donnée à l'homme pour cette exploitation sans mesure. La Terre promise promet beaucoup.

Il paraît important de distinguer, en conclusion, d'une part une mythique empruntée à Rameau (la même influence chez Labelle et Buies), d'autre part un modèle de développement explicitement emprunté aux

20 A. Buies, Le chemin de fer du Lac-Saint-Jean, Québec, L. Brousseau, 1895.

21 Provost, La Bourse, p. 151-152. 
expériences et aux espoirs américains. Le mythe du Nord est un exemple de syncrétisme et de pragmatisme comme le continent américain seul en fait naître. La Matawinie, selon Provost, est une région qui va entrer dans le développement industriel. Les paroles du prêtre résonnent régulièrement modernes; elles ne feraient pas rougir les partisans actuels de la croissance continue.

Dans vingt ans d'ici [1882], pense encore M. Brassard, que se passera-t-il sur les rives de la Mantawa? Alors, il est permis de l'espérer, il n'y aura pas une ou deux paroisses à visiter, mais plusieurs; on y arrivera en quelques heures par un chemin de fer; de grandes industries s'y seront développées, grâce aux nombreux et puissants pouvoirs d'eau disséminés dans toute cette région; les mines et les carrières y seront exploitées, l'agriculture y sera prospère. Alors et pour toujours nous aurons acquis à notre peuple un terrain propice et favorable .... ${ }^{22}$

22 Ibid., p. 230. 